\title{
Oral green tea catechin metabolites are incorporated into human skin and protect against UV radiation-induced cutaneous inflammation in association with reduced production of pro-inflammatory eicosanoid 12-hydroxyeicosatetraenoic acid
}

\author{
Lesley E. Rhodes ${ }^{1 *}$, Gemma Darby ${ }^{1}$, Karen A. Massey ${ }^{2}$, Kayleigh A. Clarke ${ }^{3}$, Tristan P. Dew ${ }^{3}$, \\ Mark D. Farrar ${ }^{1}$, Susan Bennett ${ }^{1}$, Rachel E. B. Watson ${ }^{1}$, Gary Williamson ${ }^{3}$ and Anna Nicolaou ${ }^{2}$ \\ ${ }^{1}$ Photobiology Unit, Dermatology Centre, Institute of Inflammation and Repair, University of Manchester, Manchester \\ Academic Health Science Centre, Salford Royal NHS Foundation Hospital, Manchester M6 8HD, UK \\ ${ }^{2}$ School of Pharmacy and Centre for Skin Sciences, School of Life Sciences, University of Bradford, Bradford, UK \\ ${ }^{3}$ School of Food Science and Nutrition, University of Leeds, Leeds, UK \\ (Submitted 20 September 2012 - Final revision received 26 November 2012 - Accepted 26 November 2012 - First published online 28 January 2013)
}

\begin{abstract}
Green tea catechins (GTC) reduce UV radiation (UVR)-induced inflammation in experimental models, but human studies are scarce and their cutaneous bioavailability and mechanism of photoprotection are unknown. We aimed to examine oral GTC cutaneous uptake, ability to protect human skin against erythema induced by a UVR dose range and impact on potent cyclo-oxygenase- and lipoxygenase-produced mediators of UVR inflammation, $\mathrm{PGE}_{2}$ and 12-hydroxyeicosatetraenoic acid (12-HETE), respectively. In an open oral intervention study, sixteen healthy human subjects (phototype I/II) were given low-dose GTC (540 mg) with vitamin C (50 mg) daily for $12 \mathrm{weeks}$. Preand post-supplementation, the buttock skin was exposed to UVR and the resultant erythema quantified. Skin blister fluid and biopsies were taken from the unexposed and the UVR-exposed skin $24 \mathrm{~h}$ after a pro-inflammatory UVR challenge (three minimal erythema doses). Urine, skin tissue and fluid were analysed for catechin content and skin fluid for PGE $_{2}$ and 12-HETE by liquid chromatography coupled to tandem MS. A total of fourteen completing subjects were supplement compliant (twelve female, median 42.5 years, range 29-59 years). Benzoic acid levels were increased in skin fluid post-supplementation $(P=0 \cdot 03)$, and methylated gallic acid and several intact catechins and hydroxyphenyl-valerolactones were detected in the skin tissue and fluid. AUC analysis for UVR erythema revealed reduced response post-GTC $(P=0.037)$. Pre-supplementation, $\mathrm{PGE}_{2}$ and 12 -HETE were UVR induced $(P=0 \cdot 003,0 \cdot 0001)$. After GTC, UVR-induced 12-HETE reduced from mean 64 (SD 42$)$ to 41 (SD 32) pg/ $\mu \mathrm{l}(P=0 \cdot 01)$, while PGE 2 was unaltered. Thus, GTC intake results in the incorporation of catechin metabolites into human skin associated with abrogated UVR-induced 12-HETE; this may contribute to protection against sunburn inflammation and potentially longer-term UVR-mediated damage.
\end{abstract}

Key words: Green tea catechins: Bioavailability: Skin: 12-Hydroxyeicosatetraenoic acid

UV radiation (UVR) in sunlight is a key environmental stressor that makes an impact on skin health. Acute effects include sunburn (an inflammatory response), immune suppression and photosensitivity, while repeated exposures lead to photoageing and photocarcinogenesis ${ }^{(1)}$. Sunburn is characterised clinically by erythema due to vasodilatation and, histologically, a dermal infiltrate of neutrophils and mononuclear cells is observed ${ }^{(2,3)}$. Activation of cutaneous phospholipase $A_{2}$ by UVR is a key part of the inflammatory response, releasing membrane-esterified fatty acids, including arachidonic acid, which is subsequently metabolised by cyclo-oxygenase (COX), lipoxygenase (LOX) and cytochrome $\mathrm{P} 450$ isozymes to produce eicosanoids with vasodilatory and chemoattractant properties ${ }^{(4)}$. Potent pro-inflammatory mediators, $\mathrm{PGE}_{2}$ and 12-hydroxyeicosatetraenoic acid (12-HETE), are the most abundant eicosanoids at the peak of the sunburn response, correlating with UVR upregulated expression of COX-2 and 12-LOX in human skin ${ }^{(4)}$.

Polyphenols are plant-derived molecules, many exhibiting anti-inflammatory properties ${ }^{(5,6)}$. Their oral intake is associated with health benefits, including reduced risk of cancer

Abbreviations: 12-HETE, 12-hydroxyeicosatetraenoic acid; COX, cyclo-oxygenase; EC, (-)-epicatechin; ECG, (-)-EC-3-O-gallate; EGC, (-)epigallocatechin; EGCG, (-)-EGC-3-O-gallate; GTC, green tea catechins; LOX, lipoxygenase; M4, 5-(3',4',5'-trihydroxyphenyl)- $\gamma$-valerolactone; M6, 5-(3', $4^{\prime}$-dihydroxyphenyl)-valerolactone; M6', 5-(3',5'-dihydroxyphenyl)-valerolactone; MED, minimal erythema dose; UVR, UV radiation.

* Corresponding author: Professor L. E. Rhodes, fax + 44161 2061156, email lesley.e.rhodes@manchester.ac.uk 
and $\operatorname{CVD}^{(7,8)}$. Studies performed largely in experimental models suggest that polyphenols from various sources may protect the skin against the adverse effects of UVR, including carcinogenesis $^{(1,9,10)}$. Green tea is widely consumed worldwide and contains several polyphenols of the catechin family, i.e. green tea catechins (GTC), principally (-)-epicatechin (EC), (-)-EC-3-O-gallate (ECG), (-)-epigallocatechin (EGC) and (-)-EGC-3-O-gallate $\left(\mathrm{EGCG}^{(11)}\right)$. Emerging evidence suggests that GTC can protect against cutaneous damage. Specifically, oral GTC protected against UVR-induced skin inflammation and carcinogenesis in hairless mice ${ }^{(12)}$, whilst in human subjects, topically applied GTC reduced UVR-induced DNA damage, erythema and leucocytic infiltrate $e^{(13,14)}$, and oral green tea extract reduced skin erythema following a UVR challenge near the sunburn threshold ${ }^{(15)}$. Some of these effects may be mediated via effects on COX and LOX isozymes, as EGCG, EGC, ECG and EC have been reported to reduce the production of $\mathrm{PGE}_{2}$ and/or 12-HETE in experimental systems ${ }^{(16-18)}$ and oral GTC to reduce UVRinduced COX-2 protein expression and $\mathrm{PGE}_{2}$ production in mouse epidermis ${ }^{(10)}$. However, it is unknown whether these findings have relevance to human skin.

Despite increasing evidence of their photoprotective potential, there is a dearth of information on cutaneous bioavailability of oral GTC in human subjects, reflecting the challenges of their tissue assessment. Moreover, the molecular mechanism(s) underlying protection from UVR-induced inflammation are unexplored in human subjects. Potentially, this may be conveyed through an impact on key COX- and LOX-derived pro-inflammatory eicosanoids mediating the sunburn response, which additionally exhibit promoting properties in skin carcinogenesis ${ }^{(4,19,20)}$. Thus, the aims of the present novel study were to examine directly in human subjects in vivo for evidence of cutaneous uptake of orally administered GTC, to evaluate for impact of GTC on sunburn over a range of pro-inflammatory UVR doses and to explore whether the underlying mechanism of protection could be GTC modulation of $\mathrm{PGE}_{2}$ and/or 12-HETE formation.

\section{Methods}

\section{Subjects and study design}

This was an open oral intervention study conducted in the Photobiology Unit, Dermatology Centre, Salford Royal NHS Foundation Hospital, Manchester, UK. Subjects ( $n$ 16) were white Caucasian males and females of sun-reactive skin type I-II (easy sunburn, minimal tanning). The exclusion criteria were: history of skin cancer or a photosensitivity disorder, use of a sunbed or sunbathing in the 3 months prior to the study, taking photoactive medication or nutritional supplements, consuming more than two cups of tea per $d$ and currently pregnant or breastfeeding. Ethical approval was obtained from the North Manchester Research Ethics Committee (reference 08/H1006/79). Written informed consent was obtained from the participants, and the study adhered to the Declaration of Helsinki principles.

\section{Dietary supplements}

Subjects took oral supplements comprising $540 \mathrm{mg}$ GTC with $50 \mathrm{mg}$ vitamin $\mathrm{C}$ daily for 12 weeks. These were in the form of three capsules, each containing $450 \mathrm{mg}$ green tea extract (total $1350 \mathrm{mg}$ tea, $540 \mathrm{mg}$ GTC; Table 1) and two capsules each containing $25 \mathrm{mg}$ vitamin C (total $50 \mathrm{mg}$ vitamin C), and were taken with breakfast each morning. The low-dose vitamin $\mathrm{C}$ was added to stabilise the green tea extract in the gut lumen ${ }^{(21)}$; oral vitamin $\mathrm{C}$ supplementation alone has been shown to have no impact on UVR erythema ${ }^{(22)}$. Supplements were provided by Nestec Limited and packaged by Laboratoire LPH. Compliance was assessed by counting the residual capsules in the dispensed containers that the volunteers were asked to return and through analysis of $24 \mathrm{~h}$ urine samples collected from all volunteers before and after $1 \mathrm{~d}, 6$ weeks and 12 weeks supplementation.

\section{UV radiation exposure}

UVR exposures were performed using a solar simulator, with emission of UVB and UVA mimicking that of sunlight (emission 290-400 nm; Newport Spectra-Physics Limited). Irradiance of the light source was measured $10 \mathrm{~cm}$ from the source prior to each irradiation, using a radiometer (model IL 730A; International Light) calibrated for use with the light source, to ensure consistency of the doses applied. The minimal erythema dose (MED) of UVR of each subject was assessed at baseline and post-supplementation, following application of a geometric series of ten doses of solar-simulated UVR (erythemally weighted doses $6.6-68 \mathrm{~mJ} / \mathrm{cm}^{2}$ ) to the upper buttock skin ( $1 \mathrm{~cm}$ diameter circular sites). Irradiated sites were examined visually after $24 \mathrm{~h}$, with the MED defined as the lowest dose producing visually discernible erythema. Erythema at each site was quantified as described in the following section. At $24 \mathrm{~h}$ prior to skin tissue and blister fluid sampling, doses of UVR of $3 \times$ the individual's pre-supplementation MED were given to sites on one buttock; this dose was selected in order to provoke an inflammatory response sufficient to significantly elevate cutaneous eicosanoid levels ${ }^{(4)}$.

Table 1. Catechin and gallic acid content of green tea extract* (Mean values and standard deviations)

\begin{tabular}{lrc} 
& \multicolumn{2}{c}{ Content (mg/450 mg capsule) } \\
\cline { 2 - 3 } GTC & Mean & SD \\
\hline Gallic acid & 0.4 & 0.0 \\
Catechin & 2.1 & 0.0 \\
Epicatechin & 12.5 & 0.2 \\
Gallocatechin & 12.4 & 0.6 \\
Epigallocatechin & 49.3 & 3.9 \\
Catechin gallate & 0.3 & 0.0 \\
Epicatechin gallate & 26.0 & 0.2 \\
Gallocatechin gallate & 4.5 & 0.4 \\
Epigallocatechin gallate & 72.6 & 3.1 \\
Total & 180.0 & 8.3 \\
\hline
\end{tabular}

GTC, green tea catechins.

${ }^{*}$ Contents of three capsules were homogenised and extracted in triplicate. 


\section{Quantification of the UV radiation-induced erythemal responses}

The intensity of erythema (erythema index) was quantified using a reflectance instrument (Diastron) in ten subjects. Readings were taken in triplicate from each exposed site and from adjacent unexposed skin, and erythema expressed as the difference between these readings $(\Delta E)$. Dose-response modelling was performed using a dedicated data analysis package (Regional Medical Physics Department, Gateshead \& Tyneside Health Authority, UK) to calculate each subject's $\mathrm{D}_{30}$, the UVR dose producing a $\Delta E$ of 30 arbitrary units, a threshold value that approximates an individual's visual MED.

\section{Skin biopsy and suction blister fluid sampling}

UVR-exposed $(3 \times$ MED) and -protected areas of upper buttock skin were sampled at baseline and post-supplementation; UVR exposures were limited to one buttock and the other buttock provided the unexposed skin and blister fluid samples. Skin punch biopsies ( $5 \mathrm{~mm}$ diameter) were taken after intradermal injection of lignocaine, as described ${ }^{(4)}$, snap-frozen and stored at $-80^{\circ} \mathrm{C}$. Suction blisters were raised using suctions cups with a central aperture diameter of $1 \mathrm{~cm}$ and vacuum of $250 \mathrm{mmHg}$, as described previously ${ }^{(4)}$. Skin blister fluid was aspirated with a twenty-three-gauge needle, snapfrozen in liquid $\mathrm{N}_{2}$ and stored at $-80^{\circ} \mathrm{C}$ until analysis. Samples destined for polyphenol analysis were combined with $25 \mu \mathrm{l}$ $\mathrm{NaH}_{2} \mathrm{PO}_{4}(0.4 \mathrm{~mol} / 1, \mathrm{pH} 3.6)$ containing $200 \mathrm{~g} / 1$ ascorbic acid and $1 \mathrm{~g} / \mathrm{l}$ EDTA, prior to freezing.

\section{Eicosanoid analysis}

Eicosanoids in skin blister fluid were analysed by liquid chromatography coupled to electrospray ionisation tandem MS, as described previously ${ }^{(23,24)}$. In summary, skin fluid samples (typically 50-200 $\mu \mathrm{l}$ ) were diluted with methanol-water $(15 \%, \mathrm{w} / \mathrm{w})$ up to $3 \mathrm{ml}$. Internal standards (40 ng $\mathrm{PGB}_{2}-d_{4}$ and $80 \mathrm{ng}$ 12-HETE- $d_{8}$; Cayman Chemicals) were then added and the resultant solutions acidified to $\mathrm{pH} 3 \cdot 0$, followed by solid-phase extraction (C18-E cartridges; Phenomenex), to reduce matrix effects and semi-purify the lipid mediators. Eicosanoids were analysed on a C18 column (Luna $5 \mu \mathrm{m}$; Phenomenex) using a Waters Alliance 2695 HPLC pump coupled to a triple-quadrupole mass spectrometer equipped with an electrospray ionisation probe (Quattro Ultima; Waters). Instrument control and data acquisition were performed using MassLynx 4.0 software (Waters). The following multiple reaction monitoring transitions were used for the assay: $\mathrm{PGE}_{2} \mathrm{~m} / \mathrm{z}$ $351>271 ; 12$-HETE $m / z 319>179$.

\section{Polyphenol analysis of urine, skin tissue and blister fluid}

Urine was collected in HCl-washed flasks containing ascorbate (approximately $1 \mathrm{~g} / \mathrm{l}$ ) and stored in aliquots at $-80^{\circ} \mathrm{C}$. Blister fluid and urine samples were enzymatically hydrolysed in line with previous literature ${ }^{(25)}$ with adjustments. Following thawing at $5^{\circ} \mathrm{C}$, urine was adjusted to $\mathrm{pH} 5.0$ with $\mathrm{NaOH}$
$(0 \cdot 1 \mathrm{~mol} / \mathrm{l})$. A $40 \mu \mathrm{l}$ aliquot of urine or blister fluid was combined with $4 \mu \mathrm{l} \mathrm{NaH}{ }_{2} \mathrm{PO}_{4}$ solution ( $0.4 \mathrm{~mol} / \mathrm{l}$, pH 5.0) containing $200 \mathrm{~g} / \mathrm{l}$ ascorbic acid and $1 \mathrm{~g} / \mathrm{l}$ EDTA, and $20 \mu \mathrm{l}$ sodium acetate buffer $(0 \cdot 2 \mathrm{~mol} / \mathrm{l}, \mathrm{pH} 5 \cdot 0)$ containing $0.012 \mu \mathrm{g}$ taxifolin internal standard (Extrasynthese) and $5 \mathrm{U}$ (1.39 nkat) sulphatase (Type VIII; Sigma). Based on previous optimisation work, 100 and $200 \mathrm{U}$ (0.087 and 0.175 nkat) $\beta$-glucuronidase (Type X; Sigma) in $\mathrm{NaH}_{2} \mathrm{PO}_{4}(75 \mathrm{mmol} / \mathrm{l}, \mathrm{pH}$ 6.8) were added to the blister and urine samples, respectively, and incubated at $37^{\circ} \mathrm{C}$ for 45 and $60 \mathrm{~min}$, respectively. Samples were extracted with $3 \times 250 \mu$ l ethyl acetate, with vortexing and centrifugal separation at each step. The combined extracts were dried under $\mathrm{N}_{2}$ and frozen at $-80^{\circ} \mathrm{C}$. Samples and reagents were handled on ice throughout extraction. Dried samples were reconstituted with $12 \mu \mathrm{l}$ of $20 \%$ (v/v) acetonitrile containing $1 \mathrm{~g} / 1$ ascorbic acid, and sealed in a microwell plate before analysis. With the exception of hippuric acids (which were poorly partitioned into ethyl acetate), the average extraction efficiency for catechins and phenolic acids reported (Table 2) was 84.7 (SD 13.0)\%, whilst internal standard extraction efficiency was consistently at $100 \%$.

Polyphenol conjugates required extraction from biopsy tissue before enzyme hydrolysis. Additionally, Chu et al. ${ }^{(26)}$ highlighted problems using traditional ascorbate/EDTA solutions to stabilise catechins when handling tissue, owing to intrinsic Fe content, and proposed the use of sodium dithionite, a reducing agent that does not take part in Fenton reactions. Biopsies were thawed at room temperature immediately before extraction and then kept on ice throughout the procedure. Biopsies were washed in hexane to remove blood residue. A section of dermis was separated with a scalpel and weighed. To this, $250 \mu \mathrm{l} \mathrm{N}$-flushed chloroform containing $0 \cdot 1 \mathrm{~g} / \mathrm{l}$ butylated hydroxytoluene and $250 \mu \mathrm{l}$ sodium dithionite $(0.3 \mathrm{~mol} / \mathrm{l})$ in sodium acetate buffer $(0.2 \mathrm{~mol} / 1, \mathrm{pH} 5 \cdot 0)$ were added. Samples were homogenised (Turrax micro homogenizer; IKA), with the sample being returned to ice at regular intervals, then vortexed and separated by centrifugation. The aqueous layer was removed and a second $250 \mu \mathrm{l}$ aliquot of sodium dithionate in sodium acetate buffer added for a repeat extraction. Excess chloroform was removed via $\mathrm{N}_{2}$ drying, and the combined extracts mixed with $50 \mu$ l sodium acetate buffer $(0 \cdot 2 \mathrm{~mol} / \mathrm{l}, \mathrm{pH} 5 \cdot 0)$ containing $0 \cdot 012 \mu \mathrm{g}$ taxifolin internal standard, $10 \mathrm{U}$ (2.78 nkat) sulphatase and $200 \mathrm{U}$ (0.175 nkat) $\beta$-glucuronidase. After $60 \mathrm{~min}$ incubation at $37^{\circ} \mathrm{C}$, the extraction proceeded as for blisters/urine using $3 \times 400 \mu$ l ethyl acetate.

Samples were analysed using an Agilent 1200 SL HPLC system (Agilent Technologies), which comprised a binary pump, degasser, well plate autosampler $\left(5^{\circ} \mathrm{C}\right)$ and column oven $\left(35^{\circ} \mathrm{C}\right)$ connected to a 6410 triple quadrupole LC-MS/MS. A $5 \mu \mathrm{l}$ aliquot was injected onto a Kinetex C18 microbore column $(2.6 \mu \mathrm{m}, 150 \times 2.1 \mathrm{~mm}$; Phenomenex $)$ running a binary gradient of LC-MS-grade water (Millipore) $v$. acetonitrile (Fisher), both with $0 \cdot 2 \%(\mathrm{v} / \mathrm{v})$ formic acid, at $0.3 \mathrm{ml} / \mathrm{min}$. The gradient started at $5 \%$ acetonitrile for first $5.8 \mathrm{~min}$, rose to $30 \%$ over $29.2 \mathrm{~min}$ and then increased to $95 \%$ acetonitrile over $2.4 \mathrm{~min}$. This was held for a further $3.6 \mathrm{~min}$ to wash the column and then returned to $5 \%$ acetonitrile over $3.6 \mathrm{~min}$, re-equilibrating over a further $10.9 \mathrm{~min}$. 
Table 2. Green tea catechins and their metabolites in urine at baseline and post-supplementation (Mean values and standard deviations, $n$ 13)

\begin{tabular}{|c|c|c|c|c|c|c|c|c|}
\hline \multirow[b]{3}{*}{ Compound } & \multicolumn{8}{|c|}{ Amount excreted in urine $(\mu \mathrm{mol})$} \\
\hline & \multicolumn{2}{|c|}{ Baseline } & \multicolumn{2}{|c|}{ Day 1} & \multicolumn{2}{|c|}{ Week 6} & \multicolumn{2}{|c|}{ Week 12} \\
\hline & Mean & SD & Mean & SD & Mean & SD & Mean & SD \\
\hline $\mathrm{EC} \dagger$ & 0.3 & 0.4 & $7^{\star}$ & 4 & $5^{\star}$ & 4 & $7^{\star}$ & 5 \\
\hline 3'-O-Methyl EC† & 0.06 & 0.08 & $0.6^{*}$ & 0.3 & $0.5^{\star}$ & 0.4 & $0 \cdot 6^{*}$ & 0.3 \\
\hline 4'-O-Methyl EC† & 0.04 & 0.05 & $0.2^{* \star}$ & 0.2 & $0.2^{* *}$ & 0.2 & $0.3^{*}$ & 0.2 \\
\hline ECG & 0.000 & 0.002 & $0.01^{*}$ & 0.01 & $0.02^{*}$ & 0.01 & $0.01^{\star *}$ & 0.01 \\
\hline $\mathrm{EGC}+$ & 0.2 & 0.4 & $22^{*}$ & 13 & $20^{*}$ & 16 & $25^{\star}$ & 20 \\
\hline $3^{\prime}-O$-Methyl EGC† & 0.01 & 0.04 & $0.2^{*}$ & 0.1 & $0.2^{*}$ & 0.2 & $0.2^{*}$ & 0.2 \\
\hline $4^{\prime}$-O-Methyl EGC† & 0 & 0 & $8^{* \star}$ & 8 & $8^{\star *}$ & 9 & $9^{\star \star}$ & 8 \\
\hline EGCG & 0.00 & 0.01 & $0.06^{*}$ & 0.05 & $0.06^{*}$ & 0.04 & $0.08^{* *}$ & 0.09 \\
\hline Catechin & 0.01 & 0.02 & $0 \cdot 2^{*}$ & $0 \cdot 1$ & $0 \cdot 1^{*}$ & $0 \cdot 1$ & $0.2^{*}$ & 0.2 \\
\hline Gallocatechin & 0 & 0 & $0.4^{\star \star}$ & 0.5 & $0.3^{\star \star \star}$ & 0.5 & $0.6^{\star \star}$ & 0.6 \\
\hline Gallocatechin gallate & 0 & 0 & 0.003 & 0.009 & 0 & 0 & $0.01^{*}$ & 0.02 \\
\hline Gallic acid & 0.6 & 0.7 & 1 & 1 & $0.7^{\star \star \star}$ & 0.5 & $1^{\star \star}$ & 1 \\
\hline 3-O-Methyl gallic acid & 0.6 & 0.6 & 1 & 1 & 0.9 & 0.8 & $1^{\star \star \star}$ & 1 \\
\hline 3-Hydroxybenzoic acid & 1 & 1 & 2 & 2 & 2 & 3 & $4^{\star \star \star}$ & 4 \\
\hline M4 valerolactone†‡ & 0.3 & 0.4 & 30 ** & 27 & $18^{\star \star \star}$ & 25 & $21^{\star *}$ & 21 \\
\hline M6' valerolactone $\neq$ & 0.5 & 0.7 & $18^{\star *}$ & 16 & $12^{\star *}$ & 13 & $15^{\star \star}$ & 15 \\
\hline M6 valerolactone & 10 & 12 & $33^{\star *}$ & 25 & $27^{\star \star \star}$ & 28 & $31^{\star \star \star}$ & 24 \\
\hline Syringic acid & 2 & 1 & 4 & 5 & 3 & 2 & $4^{\star \star \star}$ & 4 \\
\hline Benzoic acid & 81 & 83 & 95 & 60 & 101 & 132 & $140^{\star \star \star}$ & 120 \\
\hline Hippuric acid & 4000 & 2200 & 5100 & 2500 & 4300 & 1900 & $5300^{\star \star \star}$ & 1700 \\
\hline
\end{tabular}

EC, epicatechin; ECG, epicatechin-3-O-gallate; EGC, epigallocatechin; EGCG, epigallocatechin-3-O-gallate; M4, 5-(3',4 $4^{\prime}, 5^{\prime}$-trihydroxyphenyl)- $\gamma^{\prime}$-valerolactone; M6', 5-(3',5'-dihydroxyphenyl)-valerolactone; M6, 5-(3',4'-dihydroxyphenyl)-valerolactone.

Mean values were significantly different from baseline: ${ }^{\star} P<0.05,{ }^{\star \star} P<0.01,{ }^{\star \star \star} P<0.001$ (two-tailed paired $t$ test).

$\dagger$ Increased excretion of metabolite from baseline to week 12 in $100 \%$ of subjects.

$\ddagger \mathrm{M} 4$ and $\mathrm{M} 6{ }^{\prime}$ hydroxyphenyl-valerolactone calculated as M6 equivalents.

The flow was passed into an electrospray source, with gas temperature $350^{\circ} \mathrm{C}$, flowing at 11 litres $/ \mathrm{min}$ and with a 30 pounds per square inch (psi) nebuliser pressure. Analytes were detected in negative mode using dynamic multiple reaction monitoring acquisition. Where available, analyte transmission and $\mathrm{MS}^{2}$ transition parameters were individually optimised using standards. Internal standards for EC, (+)-catechin, EGC, ECG, EGCG and taxifolin were obtained from Extrasynthese. The retention times of gallocatechin, catechin gallate and gallocatechin gallate were determined by placing aqueous solutions of the relevant epi-isomers into a boiling water-bath for $1 \mathrm{~h}$. The chromatographic method did not distinguish between $(+)$ - and (-)-enantiomers. The $3^{\prime}$ and $4^{\prime}$ monomethylated forms of EC and EGC were obtained from Nacalai Tesque. Benzoic acid, 3-hydoxy benzoic acid, hippuric acid, 3,4-dihydroxyphenylacetic acid and 3-(2',4'dihydroxyphenyl) propionic acid were obtained from Fluka and 4-hydroxy benzoic acid from Aldrich. Vanillic acid, 3,5 dihydroxy benzoic acid, gallic acid, syringic acid, 3- and 4-hydroxyphenyl acetic acids and 3-(3'-hydroxyphenyl)-propionic acid were obtained from Alfa Aesar. 3- and 4-Methyl gallic acids were obtained from Apin Chemicals, and 2,4-dihydroxy benzoic acid, 2,4,6-trihydroxy benzoic acid, 2-hydroxyphenyl acetic acid and 2-hydroxy hippuric acid from Acros Organics. All standards were of HPLC quality ( $>95 \%$ purity). As commercial standards for hydroxyphenyl-valerolactones were not available, these were tentatively identified using previously reported $\mathrm{MS}^{2}$ fragment patterns ${ }^{(27)}$. Analyte transmission and quantifying/qualifying $\mathrm{MS}^{2}$ transition parameters were individually optimised using repeat injections of extracted urine.
A total of three hydroxyphenyl-valerolactones were followed, namely, 5-(3', $4^{\prime}, 5^{\prime}$-trihydroxyphenyl)- $\gamma$-valerolactone (M4; $\mathrm{m} / z$ $223>179+138), \quad 5$ - $\left(3^{\prime}, 4^{\prime}\right.$-dihydroxyphenyl)-valerolactone (M6; $m / z \quad 207>163+122)$ and 5-(3',5'-dihydroxyphenyl)valerolactone $\left(\mathrm{M} 6^{\prime} ; m / z 207>163+123\right)$. M6 $v$. M6 $6^{\prime}$ retention time was differentiated using a synthetic M6 standard ${ }^{(28)}$, which was used to quantify all hydroxyphenyl-valerolactones. Following peak integration, peak areas were normalised to the internal standard. Whilst response factors for hippuric and benzoic acids were low (on column limit of quantification of 3.45 and $50 \mathrm{pmol}$, respectively), the universally high levels of these compounds in urine, skin fluid and tissue meant that quantification was achievable. The average on column limit of quantification for all other compounds was 380 (SD 365) fmol.

\section{Statistical analysis}

Parametric data were tested using the paired $t$ test. The Wilcoxon signed-rank test was used for data not satisfying assumptions of normality. Analyses were performed using StatsDirect (version 2.7.7, StatsDirect Limited). Statistical significance was accepted at $P<0 \cdot 05$. Data are shown as means and standard deviations and presented graphically as means with their standard errors.

\section{Results}

\section{Study subjects and compliance}

Of the sixteen subjects recruited to the study, one withdrew before completion for reasons unrelated to the study. 
The supplement was well-tolerated; four subjects reported mild nausea following its ingestion. Post-supplementation, all four major EC and their metabolites were present in the urine at day 1 and weeks 6 and 12 from fourteen of the fifteen subjects completing the study (Table 2). Thus, one subject was non-compliant and fourteen subjects (twelve female), with a median age of 42.5 years (range 29-59 years), were included in the study analyses.

\section{Urinary metabolites}

Of the thirty-five tea phenolics and metabolites investigated, $t$ test analysis showed that twenty components were significantly higher in week 12 urine samples compared with baseline $(P<0.05 ; n 13$ due to absent record of one sample volume; Table 2), whilst eight of these were consistently higher in all participants. As well as several intact catechins, gallic acid and methylated metabolites, hydroxyphenyl-valerolactones, benzoic acid and its glycine conjugate, hippuric acid, were all increased in the urine following GTC consumption. Based on a daily intake of $129.2 \mu \mathrm{mol}$ of EC and $482.9 \mu \mathrm{mol}$ of EGC, average urine excretion of all intact EC and EGC metabolites (including methylated forms) represented $6 \cdot 1$ and $7 \cdot 1 \%$ of the dose, respectively.

\section{Skin uptake}

Skin fluid and biopsy (dermal) samples were taken from a subgroup of ten participants at baseline and week 12, and subjected to qualitative analysis (Table 3). A total of twenty different phenolic compounds were observed in both sample types following supplementation. In blister fluid, hippuric, benzoic and 4-hydroxybenzoic acids were consistently present in all ten participants. Interestingly, methylated gallic

Table 3. Presence of green tea catechins and their metabolites in skin blister fluid and tissue samples post-supplementation (week 12; $n$ 10)†

\begin{tabular}{|c|c|c|c|c|}
\hline \multirow[b]{2}{*}{ Compound } & \multicolumn{2}{|c|}{ Skin blister fluid } & \multicolumn{2}{|c|}{ Skin biopsy } \\
\hline & $\begin{array}{c}\text { Change } \\
\text { from } \\
\text { average } \\
\text { baseline } \\
\text { value }\end{array}$ & $\begin{array}{l}\text { Detected } \\
\text { in } n \\
\text { participants }\end{array}$ & $\begin{array}{c}\text { Change } \\
\text { from } \\
\text { average } \\
\text { baseline } \\
\text { value }\end{array}$ & $\begin{array}{l}\text { Detected } \\
\text { in } n \\
\text { participants }\end{array}$ \\
\hline Benzoic acid & $+36 \%{ }^{*}$ & 10 & ND & 10 \\
\hline 4-OH-Benzoic acid & ND & 10 & ND & 10 \\
\hline Hippuric acid & ND & 10 & ND & 6 \\
\hline 4-O-Me-gallic acid & ND & 5 & ND & 2 \\
\hline $\mathrm{EC}$ & - & - & PPS & 2 \\
\hline EGC & PPS & 2 & PPS & 1 \\
\hline EGC-4-Me & - & - & PPS & 4 \\
\hline EGCG & - & - & PPS & 1 \\
\hline M4 valerolactone & PPS & 2 & - & - \\
\hline M6 valerolactone & PPS & 2 & - & - \\
\hline
\end{tabular}

ND, no significant difference; EC, epicatechin; PPS, only present postsupplementation; EGC, epigallocatechin; EGCG, epigallocatechin-3-O-gallate; M4, 5-(3', 4',5'-trihydroxyphenyl)- $\gamma$-valerolactone; M6, 5-(3', $4^{\prime}$-dihydroxyphenyl)valerolactone.

*Value was significantly different compared with baseline $(P=0.03$, two-tailed paired $t$ test).

$\dagger$ Paired $t$ test performed only for compounds present in all subjects.

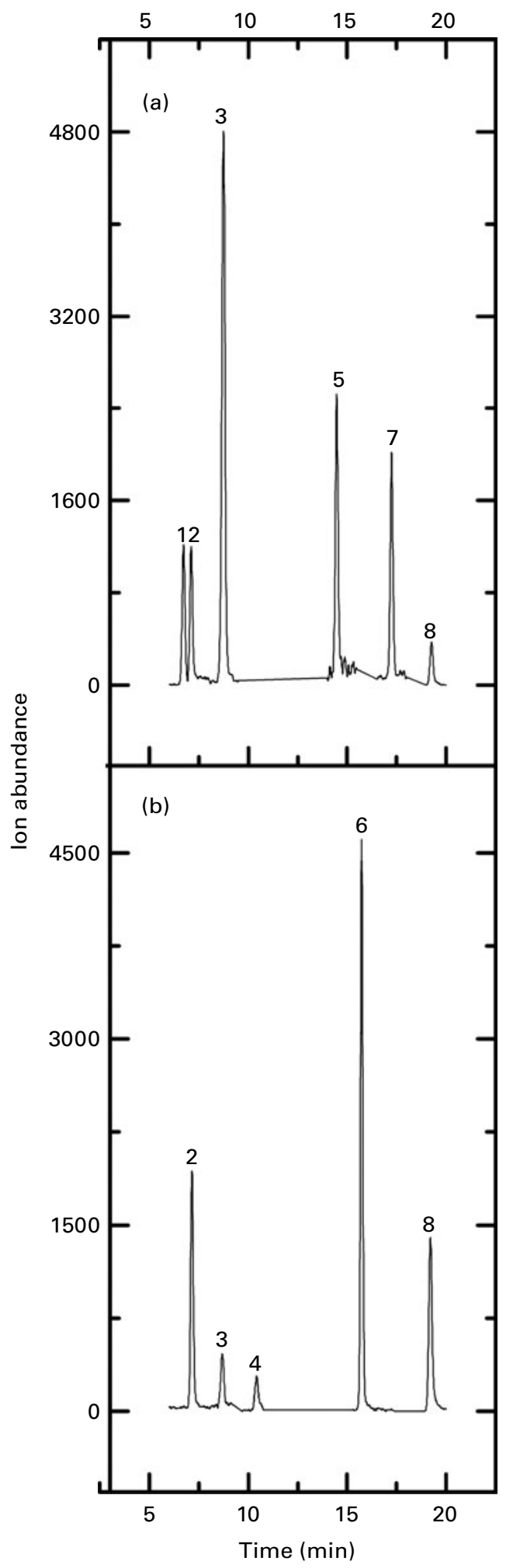

Fig. 1. Liquid chromatography-MS/MS total ion current chromatogram of (a) major compounds in skin fluid and (b) dermal skin tissue extract post-green tea catechin supplementation (week 12). Peak identities and multiple reaction monitoring $\mathrm{m} / \mathrm{z}$ transitions are: 1, M4 hydroxyphenyl-valerolactone $(223>179) ; 2$, 4-hydroxybenzoic acid (137>93); 3, hippuric acid $(178>134) ; 4$, 2,4-dihydroxybenzoic acid (153 > 109); 5, M6 hydroxyphenyl-valerolactone $(207>163)$; 6 , epicatechin $(289>245)$; 7, 3-(3'-hydroxyphenyl)-propionic acid (165 > 121); and 8, benzoic acid (121>77). 
acid and several intact catechins and catechin ring-fission products were also observed, with 4-O-methyl gallic acid present in half of the subjects, and EGC, M4 and M6 hydroxyphenyl-valerolactones observed in fluid from two participants (Fig. 1). Change from baseline was only statistically significant for benzoic acid $(P=0 \cdot 03)$. Benzoic acid and its 4-hydroxylated form were also detected in all biopsy samples, whilst hippuric acid was only observed in six volunteers. Following supplementation, 4'-O-methylated EGC ( $n$ 4), EGC ( $n$ 1), EC ( $n$ 2), EGCG ( $n$ 1) and 4-O-methyl gallic acid ( $n$ 2) were observed in the dermis of certain volunteers.

\section{UV radiation erythema dose-response}

The median MED was $35 \mathrm{~mJ} / \mathrm{cm}^{2}$ at baseline and this was unchanged post-supplementation. Dose-response analysis showed a small increase in $\mathrm{D}_{30}$ from a mean of 28.0 (sD 7.7$) \mathrm{mJ} / \mathrm{cm}^{2}$ at baseline to $32.9(\mathrm{sD} 11.0) \mathrm{mJ} / \mathrm{cm}^{2}$ postsupplementation, although this did not reach statistical significance $(P=0 \cdot 17)$. However, GTC supplementation resulted in a significant decrease in erythema at the maximum UVR dose given $\left(68 \mathrm{~mJ} / \mathrm{cm}^{2}\right.$ erythemally weighted UVR), with $\Delta E$ falling from 100.2 (SD 21.4) at baseline to $81 \cdot 2$ (SD 23.2) post-supplementation ( $P=0.006$; Fig. 2(a)). AUC analysis of the UVR erythema dose-response showed a significant reduction in the erythema response post-supplementation $(P=0 \cdot 037$; Fig. 2(b)).

\section{Production of $P G E_{2}$}

Pre-supplementation, mean concentration of $\mathrm{PGE}_{2}$ in blister fluid from unexposed skin was 49.1 (SD 34.9) pg/ $\mu$ l. Production of $\mathrm{PGE}_{2}$ significantly increased by approximately 2.3-fold following exposure to $3 \times$ MED UVR $(P=0.003$; Fig. 3(a)). Post-supplementation, $\mathrm{PGE}_{2}$ in unexposed skin was similar to baseline $(47.5(\mathrm{SD} 30.5) \mathrm{pg} / \mu \mathrm{l})$. Exposure to the same UVR dose as at baseline produced a significant rise in $\mathrm{PGE}_{2}$ (approximately 2.4-fold; $P=0.001$ ), with no significant difference in $\mathrm{PGE}_{2}$ concentration between exposed skin at baseline and post-supplementation.

\section{Production of 12-hydroxyeicosatetraenoic acid}

Pre-supplementation, the concentration of 12-HETE was significantly approximately five-fold higher in UVR-exposed skin compared with unexposed skin $(P=0 \cdot 0001)$. Following supplementation, the UVR-induced rise in 12-HETE was approximately $2 \cdot 7$-fold ( $P=0 \cdot 004$; Fig. $3(\mathrm{~b})$ ), with significantly lower concentration of 12-HETE in UVR-exposed skin compared with the baseline $(P=0 \cdot 01)$, and no significant difference in unexposed skin.

\section{Discussion}

The present human oral intervention study is novel in several respects: it evaluates cutaneous uptake of catechins and catechin metabolites, measures the impact of low-dose green tea supplementation on pro-inflammatory UVR challenges to the
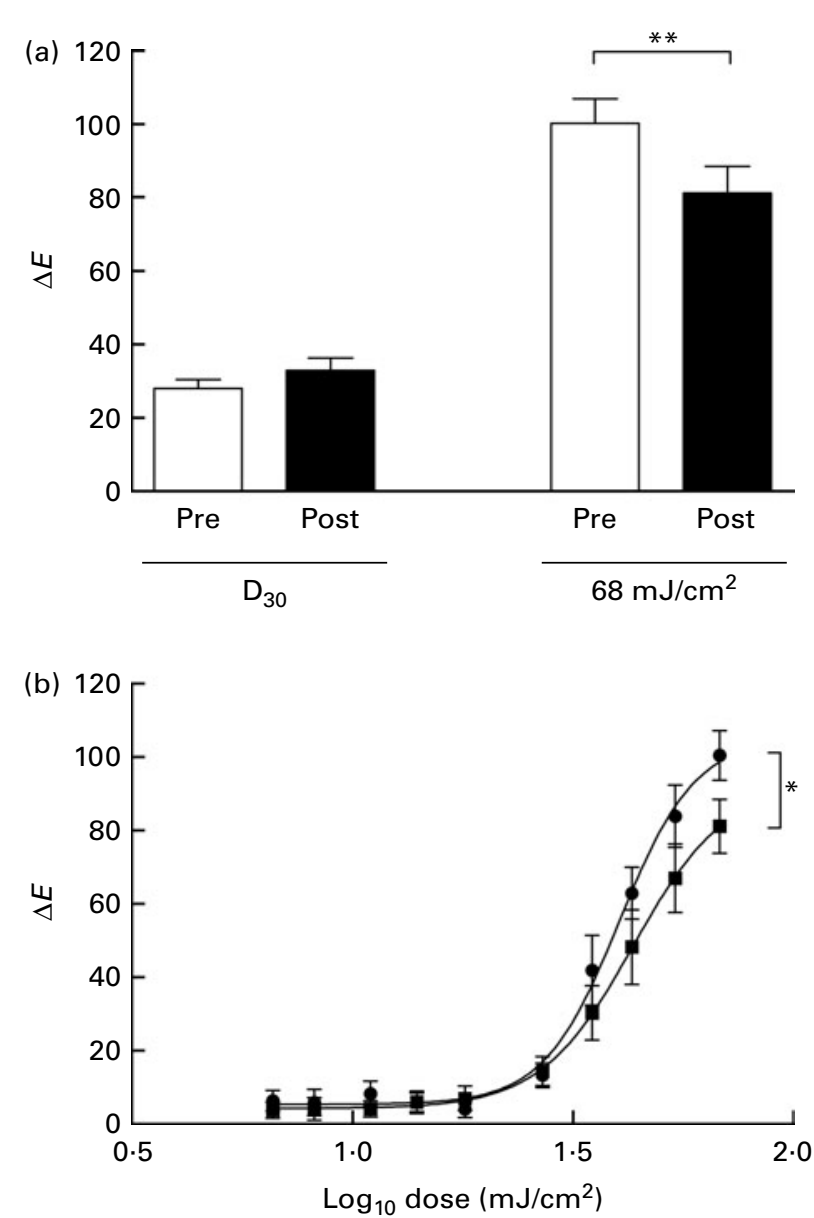

Fig. 2. Impact of oral green tea catechins on UV radiation-induced erythema. (a) Erythema response to solar-simulated UV radiation at the $D_{30}$ and the highest dose $\left(68 \mathrm{~mJ} / \mathrm{cm}^{2}\right)$, before and after 12 weeks supplementation. (b) UV radiation erythema dose-response curves before $(\bullet)$ and after (ם) 12 weeks supplementation. Values are means, with their standard errors represented by vertical bars $(n 10)$. Mean values were significantly different: ${ }^{\star} P<0.05,{ }^{* *} P<0.01$ (two-tailed paired $t$ test).

skin and examines the potential for protection through reduction of pro-inflammatory eicosanoid production. Our data provide the first evidence that GTC can be taken up into the skin following oral intake in human subjects and indicate their complex skin incorporation pattern. Significant reduction was found in the cutaneous UVR erythema doseresponse, with greatest effect at higher doses, and this reduced inflammation may be attributable to the associated significant abrogation of UVR up-regulation of the potent pro-inflammatory 12-LOX metabolite, 12-HETE. In contrast, no evidence was found for mediation of the protection conferred by GTC through an impact on the COX-2 metabolite $\mathrm{PGE}_{2}$.

The finding that GTC protects against UVR-induced erythema in human subjects is supported by previous studies of its topical application ${ }^{(13,14)}$ and a recent oral study ${ }^{(15)}$. In the latter, volunteers consumed a green tea beverage providing a much higher dose of $1402 \mathrm{mg}$ catechins/d for 12 weeks, and this protected against the threshold erythema induced by the single UVR dose tested. We found a small (non-statistically significant) effect at the threshold value $D_{30}$ and demonstrated 


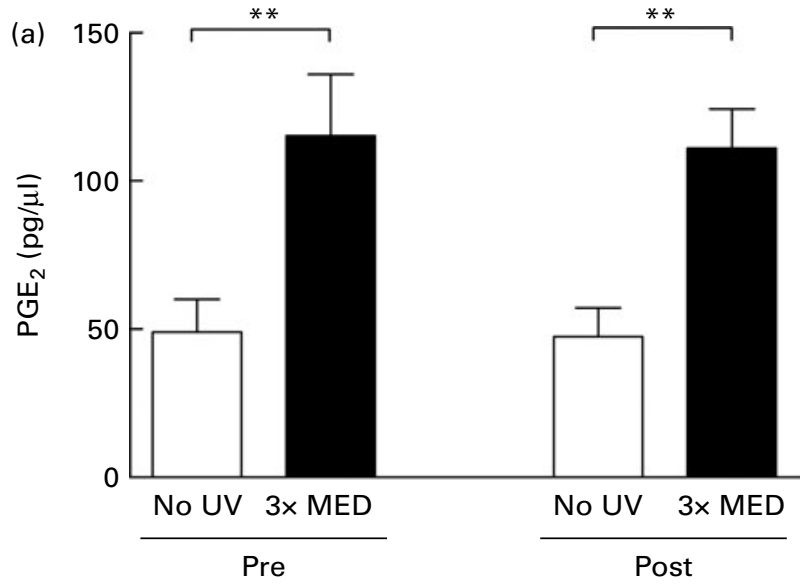

(b)

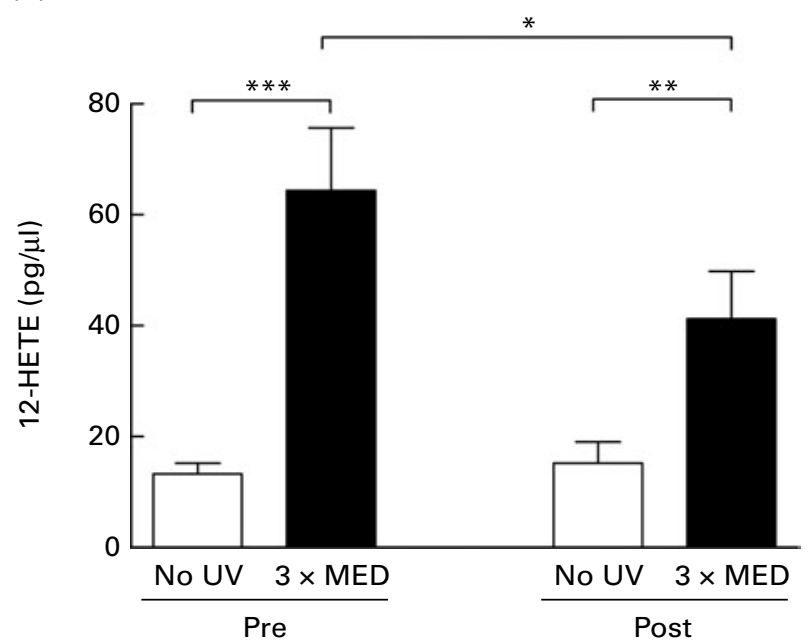

Fig. 3. Concentration of (a) $\mathrm{PGE}_{2}(n$ 10) and (b) 12-hydroxyeicosatetraenoic acid (12-HETE, $n$ 14) in skin fluid from unexposed skin and skin exposed to $3 \times$ minimal erythema dose (MED) of solar-simulated UV radiation both preand post-supplementation for 12 weeks with green tea catechins. Values are means, with their standard errors represented by vertical bars. Mean values were significantly different: ${ }^{\star} P<0.05$, ${ }^{\star \star} P<0.01$, ${ }^{\star \star *} P<0.001$ (two-tailed paired $t$ test for $\mathrm{PGE}_{2}$, Wilcoxon signed-rank test for 12-HETE).

how oral supplementation with GTC can protect against the inflammation produced over a range of higher UVR doses, such as can be achieved when individuals over-expose themselves to sunlight. Since one large cup of green tea $(250 \mathrm{ml})$ contains approximately $300 \mathrm{mg}$ of catechins (EC, ECG, EGC and EGCG), then the modest level of GTC intake in the present study, i.e. approximately $540 \mathrm{mg}$, is seen to be readily achievable in daily life, and this is already consumed in many parts of the world.

Compliance with supplement ingestion was confirmed by demonstration of the urinary content of all four major categories of catechins in GTC in all but one completing volunteer, who was then excluded. As expected, the predominant intact catechins found in urine were not gallate esters, and the bioavailability of EC and EGC was in-line with the reported studies ${ }^{(29,30)}$. GTC intervention resulted in a significant increase in the excretion of the majority of intact catechins from baseline at day 1 and throughout the 12-week study, with no apparent accumulation or adaptive response during this time. However, the excretion of several general polyphenol breakdown products, including hippuric, benzoic and syringic acids, was only significantly elevated from baseline after 12 weeks of intervention. Hippuric acid has previously been reported as the primary urinary metabolite following both green and black tea intervention, with participants excreting 3.8 ( $\mathrm{SD} 0.3$ ) and $4 \cdot 2$ (SD 0.3$) \mathrm{mmol} /$ $24 \mathrm{~h}$, respectively, following a $6 \mathrm{~g} / \mathrm{d}$ intervention with tea solids ${ }^{(31)}$. Whilst hippuric acid was indeed the major urinary metabolite detected in the present study $(5.3$ (SD 1.7$) \mathrm{mmol} /$ $24 \mathrm{~h}$ post-supplementation), its significant increase from baseline (at week 12) was only in the order of approximately $30 \%$. Hippuric acid is a terminal metabolite of benzoic acid, which itself is a colonic breakdown product common to various phenolic substances. Hippuric acid excretion is therefore not unique to GTC per se, and its use as a biomarker of catechin consumption in free-living populations is limited. Hydroxyphenyl-valerolactones are catechin metabolites produced by colonic ring fission: M4 and M6' are predominantly derived from EGC and M6 from $\mathrm{EC}^{(27)}$. Previously, Lee et al. ${ }^{(30)}$ reported M6 as accounting for $11.2 \%$ of EC dose in eight human subjects, although considerable variability was observed in M6 plasma levels. Urinary M4 was reported to account for just $1.4 \%$ of the EGC dose. In the present study, M6 accounted for approximately $24 \%$ of EC dose on average at week 12, with M4 and M6 ${ }^{\prime}$ accounting for approximately $4 \%$ and approximately $3 \%$, respectively, of the EGC dose. Levels of hydroxyphenyl-valerolactone excretion were significantly increased compared with baseline at day 1 and throughout the 12 week intervention, without a significant change in the level of excretion between acute and chronic GTC consumption. Therefore, we propose that these compounds may serve as a useful biomarker of EC and EGC intake, over both the short and long term.

Detecting polyphenols and metabolites in tissues is a challenge, as they bind to proteins, are at low levels and extraction methods are in development. We discovered that benzoic acid, its 4-hydroxyl form and its glycine-conjugate, hippuric acid, were typically present in both skin blister fluid and dermis. Wide inter-individual differences in oral bioavailability and metabolism of polyphenols in foods are commonly reported $^{(30,32)}$. Consistent with this, intact catechins, gallic acids and catechin ring-fission products were observed in the skin fluid and dermal samples of some, but not all volunteers following GTC supplementation. However, significant post-supplement increases in blister fluid benzoic acid content indicates that volunteers experienced an increase in polyphenol metabolites in the target area as a consequence of GTC intervention, at least partially derived from metabolism by colonic microflora.

The reduced inflammatory response to UVR on GTC was associated with significant reduction in UVR induction of the hydroxy fatty acid, 12-HETE, the most abundant pro-inflammatory eicosanoid induced in human skin by UVR exposure. As well as being a leucocyte chemoattractant, this potent keratinocyte-derived mediator has been shown to cause a 


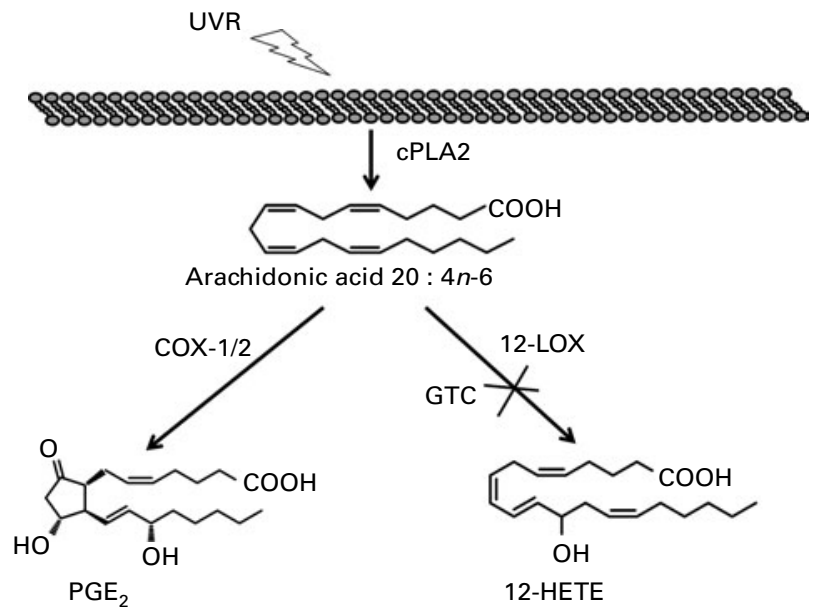

Fig. 4. Schematic to illustrate proposed mechanism of the impact of green tea catechins (GTC) and metabolites on UV radiation (UVR)-induced 12hydroxyeicosatetraenoic acid (12-HETE) production. COX, cyclo-oxygenase; $\mathrm{CPLA}_{2}$, cutaneous phospholipase $\mathrm{A}_{2} ; 12$-LOX, 12-lipoxygenase.

dose-related erythema when applied to human skin in vivo (33). While more attention has been focused on the role of $\mathrm{PGE}_{2}$ in mediating erythema, COX-2 inhibitors only partially suppress UVR erythema, whilst completely suppressing UVR-induced $\mathrm{PGE}_{2}{ }^{(34)}$ and LOX-derived mediators could also contribute ${ }^{(35)}$. Promotion of neutrophil and mononuclear cell migration into the dermis by 12-HETE may further augment the dermal vasodilatation and leucocytic infiltration through neutrophil release of vasodilatory $\mathrm{NO}$, reactive oxygen species and chemokines ${ }^{(36)}$. Other antioxidant and cell signalling activities of GTC may also contribute to reduction of UVR inflammation $^{(1,9)}$, including through modulation of transcription factor $\mathrm{NF}-\mathrm{\kappa B}^{(37)}, \mathrm{NO}^{(19,38)}$ and reduced formation/enhanced repair of UVR-induced DNA damage ${ }^{(10,14,39)}$.

Our data indicate a direct effect of oral GTC on 12-LOX and/ or possibly cytochrome $\mathrm{P} 450$ isoforms producing 12-HETE following UVR, but not on COX-2 (Fig. 4). This contrasts with studies in prostate and colon cancer cell lines, where the most abundant polyphenolic compound in tea, EGCG, inhibited protein and/or mRNA expression of COX-2 ${ }^{(40,41)}$. However, EGCG, EGC and ECG are reported to inhibit LOX activity in colonic mucosa ${ }^{(16)}$ and EC to inhibit activity of human platelet $12-\mathrm{LOX}^{(17)}$. Topical green tea polyphenols $(1-24 \mathrm{mg}$ in $200 \mu \mathrm{l}$ acetone) in mice reduced the activity of both LOX and COX enzymes after 12-O-tetradecanoylphorbol-13-acetate-induced tumour production, resulting in decreased $\mathrm{PGE}_{2}$ and 12 -HETE production ${ }^{(42)}$. Differences in findings are not unexpected between experimental models and human skin in vivo, and the catechin dose applied might also influence outcomes ${ }^{(43,44)}$.

UVR is the principal aetiological factor in the majority of skin cancers, through its actions as a tumour promoter, as well as an initiator of DNA damage that can lead to mutagenesis, and repeated acute UVR insults to the skin are a risk factor for skin cancer development. Interestingly, 12-HETE is over-expressed in a variety of human tumours, including skin cancer, and it has tumour-promoting ability, which is thought to be conveyed by its anti-apoptotic and angiogenic properties $^{(45,46)}$. Moreover, inhibitors of 12-HETE are successful in protecting against tumorigenesis in cancer cell lines ${ }^{(47)}$. This adds to the other evidence, suggesting that GTC may have potential for development as an effective and safe chemopreventive agent in human subjects, as in murine UVRinduced skin tumours ${ }^{(9)}$.

In summary, the present study indicates that following oral ingestion, GTC metabolites reach the target organ skin in human subjects, and that they suppress the biosynthesis of eicosanoid 12-HETE and sunburn erythema induced by proinflammatory UVR challenges. Manipulation of pro-inflammatory signalling pathways through supplementation with nutritional bioactives is an attractive strategy for photoprotection in human subjects and may represent a complementary approach to topical sunscreens, which are infrequently and generally poorly applied ${ }^{(48)}$. Further studies are indicated to assess 12-LOX as a molecular target of oral GTC in human skin, alongside scrutiny for their potential longer-term photoprotective benefit

\section{Acknowledgements}

This study was supported by grant BB/G005575/1 from the Biotechnology and Biological Sciences Research Council Diet and Health Research Industry Club. We thank Begonia Batolomé (Instituto de Investigación en Ciencias de la Alimentación CSIC-UAM, Madrid) for the kind provision of M6 hydroxyphenyl-valerolactone standard and Andrew Healey (Analytical Centre, University of Bradford) for excellent technical assistance. L. E. R., G. W., A. N. and R. E. B. W. designed the research; G. D., K. A. M., K. A. C., T. P. D. and S. B. conducted the research; G. D., K. A. M., K. A. C., T. P. D., M. D. F. and A. N. analysed data; L. E. R., G. D. and M. D. F. wrote the paper and all authors contributed to later drafts; L. E. R., G. W. and A. N. had primary responsibility for final content. All authors read and approved the final manuscript. None of the authors declared a conflict of interest.

\section{References}

1. Swindells K \& Rhodes LE (2004) Influence of oral antioxidants on ultraviolet radiation-induced skin damage in humans. Photodermatol Photoimmunol Photomed 20, 297-304.

2. Hawk JL, Murphy GM \& Holden CA (1988) The presence of neutrophils in human cutaneous ultraviolet-B inflammation. Br J Dermatol 118, 27-30.

3. Strickland I, Rhodes LE, Flanagan BF, et al. (1997) TNF- $\alpha$ and IL-8 are upregulated in the epidermis of normal human skin after UVB exposure: correlation with neutrophil accumulation and E-selectin expression. I Invest Dermatol 108, $763-768$.

4. Rhodes LE, Gledhill K, Masoodi M, et al. (2009) The sunburn response in human skin is characterized by sequential eicosanoid profiles that may mediate its early and late phases. FASEB J 23, 3947-3956.

5. Navarro-Peran E, Cabezas-Herrera J, Sanchez-Del-Campo L, et al. (2008) The anti-inflammatory and anti-cancer properties of epigallocatechin-3-gallate are mediated by folate 
cycle disruption, adenosine release and NF-kappaB suppression. Inflamm Res 57, 472-478.

6. Rahman I, Biswas SK \& Kirkham PA (2006) Regulation of inflammation and redox signaling by dietary polyphenols. Biochem Pharmacol 72, 1439-1452.

7. Lambert JD \& Elias RJ (2010) The antioxidant and prooxidant activities of green tea polyphenols: a role in cancer prevention. Arch Biochem Biophys 501, 65-72.

8. Hooper L, Kroon PA \& Rimm EB (2008) Flavonoids, flavonoid-rich foods, and cardiovascular risk: a meta-analysis of randomized controlled trials. Am J Clin Nutr 88, 38-50.

9. Afaq F \& Mukhtar H (2006) Botanical antioxidants in the prevention of photocarcinogenesis and photoaging. Exp Dermatol 15, 678-684.

10. Meeran SM, Akhtar S \& Katiyar SK (2009) Inhibition of UVBinduced skin tumor development by drinking green tea polyphenols is mediated through DNA repair and subsequent inhibition of inflammation. J Invest Dermatol 129, $1258-1270$.

11. Neveu V, Perez-Jimenez J, Vos F, et al. (2010) PhenolExplorer: an online comprehensive database on polyphenol contents in foods. Database (Oxford) 2010, bap024.

12. Afaq F, Ahmad N \& Mukhtar H (2003) Suppression of UVBinduced phosphorylation of mitogen-activated protein kinases and nuclear factor kappa B by green tea polyphenol in SKH-1 hairless mice. Oncogene 22, 9254-9264.

13. Katiyar SK, Matsui MS, Elmets CA, et al. (1999) Polyphenolic antioxidant (-)-epigallocatechin-3-gallate from green tea reduces UVB-induced inflammatory responses and infiltration of leukocytes in human skin. Photochem Photobiol 69, $148-153$.

14. Katiyar SK, Perez A \& Mukhtar H (2000) Green tea polyphenol treatment to human skin prevents formation of ultraviolet light B-induced pyrimidine dimers in DNA. Clin Cancer Res 6, 3864-3869.

15. Heinrich U, Moore CE, De Spirt S, et al. (2011) Green tea polyphenols provide photoprotection, increase microcirculation, and modulate skin properties of women. $J$ Nutr 141, $1202-1208$

16. Hong J, Smith TJ, Ho CT, et al. (2001) Effects of purified green and black tea polyphenols on cyclooxygenase and lipoxygenase-dependent metabolism of arachidonic acid in human colon mucosa and colon tumour tissues. Biochem Pharmacol 62, 1175-1183.

17. Schewe T, Sadik C, Klotz LO, et al. (2001) Polyphenols of cocoa: inhibition of mammalian 15-lipoxygenase. Biol Chem 382, 1687-1696.

18. Singh T \& Katiyar SK (2011) Green tea catechins reduce invasive potential of human melanoma cells by targeting COX-2, $\mathrm{PGE}_{2}$ receptors and epithelial-to-mesenchymal transition. PLoS One 6, e25224.

19. Rhodes LE, Belgi G, Parslew R, et al. (2001) Ultraviolet-Binduced erythema is mediated by nitric oxide and prostaglandin $\mathrm{E}_{2}$ in combination. J Invest Dermatol 117, 880-885.

20. Pilkington SM, Watson RE, Nicolaou A, et al. (2011) Omega-3 polyunsaturated fatty acids: photoprotective macronutrients. Exp Dermatol 20, 537-543.

21. Chen ZY, Zhu QY, Wong YF, et al. (1998) Stabilizing effect of ascorbic acid on green tea catechins. J Agr Food Chem 46, $2512-2516$.

22. McArdle F, Rhodes LE, Parslew R, et al. (2002) UVR-induced oxidative stress in human skin in vivo: effects of vitamin $\mathrm{C}$ supplementation. Free Rad Biol Med 33, 1355-1362.

23. Masoodi M \& Nicolaou A (2006) Lipidomic analysis of twenty-seven prostanoids and isoprostanes by liquid chromatography/electrospray tandem mass spectrometry Rapid Commun Mass Spectrom 20, 3023-3029.

24. Masoodi M, Mir AA, Petasis NA, et al. (2008) Simultaneous lipidomic analysis of three families of bioactive lipid mediators leukotrienes, resolvins, protectins and related hydroxy-fatty acids by liquid chromatography/electrospray tandem mass spectrometry. Rapid Commun Mass Spectrom 22, 75-83.

25. Li C, Lee MJ, Sheng S, et al. (2000) Structural identification of two metabolites of catechins and their kinetics in human urine and blood after tea ingestion. Chem Res Toxicol 13, $177-184$

26. Chu KO, Wang CC, Chu CY, et al. (2004) Determination of catechins and catechin gallates in tissues by liquid chromatography with coulometric array detection and selective solid phase extraction. J Chrom B 810, 187-195.

27. Sang S, Lee MJ, Yang I, et al. (2008) Human urinary metabolite profile of tea polyphenols analyzed by liquid chromatography/electrospray ionization tandem mass spectrometry with data-dependent acquisition. Rapid Commun Mass Spectrom 22, 1567-1578.

28. Sanchez-Patan F, Chioua M, Garrido I, et al. (2011) Synthesis, analytical features, and biological relevance of 5-( $3^{\prime}, 4^{\prime}$-dihydroxyphenyl)-gamma-valerolactone, a microbial metabolite derived from the catabolism of dietary flavan-3-ols. J Agric Food Chem 59, 7083-7091.

29. Van Amelsvoort JJM, Van Het Hof KH, Mathot JNJJ, et al. (2001) Plasma concentrations of individual tea catechins after a single oral dose in humans. Xenobiotica 31, 891-901.

30. Lee MJ, Maliakal P, Chen L, et al. (2002) Pharmacokinetics of tea catechins after ingestion of green tea and (-)-epigallocatechin-3-gallate by humans: formation of different metabolites and individual variability. Cancer Epidem Biomar 11, 1025-1032.

31. Mulder TP, Rietveld AG \& van Amelsvoort JM (2005) Consumption of both black tea and green tea results in an increase in the excretion of hippuric acid into urine. $\mathrm{Am}$ J Clin Nutr 81, 256S-260S.

32. Hong YJ \& Mitchell AE (2004) Metabolic profiling of flavonol metabolites in human urine by liquid chromatography and tandem mass spectrometry. I Agric Food Chem $\mathbf{5 2}$ 6794-6801.

33. Wollard PM, Cunnigham FM, Murphy GM, et al. (1989) A comparison of the proinflammatory effects of $12(R)$ - and 12(S)-hydroxy-5,8,10,14-eicosatetraenoic acid in human skin. Prostaglandins 38, 465-471.

34. Kozuka T, Francis DM \& Greaves MW (1983) Arachidonic acid metabolites and the skin. Ann Acad Med Singapore 12, 87-91.

35. Ruzicka T (1992) The role of the epidermal 12-hydroxyeicosatetraenoic acid receptor in the skin. Eicosanoids $\mathbf{5}$ S63-S65.

36. Dowd PM, Kobza Black A, et al. (1985) Cutaneous responses to 12-hydroxy-5,8,10,14-eicosatetraenoic acid (12-HETE). J Invest Dermatol 84, 537-541.

37. Luo D, Min W, Lin XF, et al. (2006) Effect of epigallocatechingallate on ultraviolet B-induced photodamage in a keratinocyte cell line. Am J Chin Med 34, 911-922.

38. Galleano M, Pechanova O \& Fraga CG (2010) Hypertension, nitric oxide, oxidants, and dietary plant polyphenols. Curr Pharm Biotechnol 11, 837-848.

39. Nichols JA \& Katiyar SK (2010) Skin photoprotection by natural polyphenols: anti-inflammatory, antioxidant and DNA repair mechanisms. Arch Dermatol Res 302, 71-83.

40. Hussain T, Gupta S, Adhami VM, et al. (2005) Green tea constituent epigallocatechin-3-gallate selectively inhibits COX-2 
without affecting COX-1 expression in human prostate carcinoma cells. Int J Cancer 113, 660-669.

41. Peng G, Dixon DA, Muga SJ, et al. (2006) Green tea polyphenol (-)-epigallocatechin-3-gallate inhibits cyclooxygenase-2 expression in colon carcinogenesis. Mol Carcinogenesis $\mathbf{4 5}$, 309-319

42. Katiyar SK, Agarwal R, Wood GS, et al. (1992) Inhibition of 12-O-tetradecanoylphorbol-13-acetate-caused tumor promotion in 7,12-dimethylbenz $[a]$ anthracene-initiated SENCAR mouse skin by a polyphenolic fraction isolated from green tea. Cancer Res 52, 6890-6897.

43. Ahmad N, Feyes DK, Nieminen AL, et al. (1997) Green tea constituent epigallocatechin-3-gallate and induction of apoptosis and cell cycle arrest in human carcinoma cells. J Natl Cancer Inst 89, 1881-1886.
44. Ahmad N, Gupta S \& Mukhtar H (2000) Green tea polyphenol epigallocatechin-3-gallate differentially modulates nuclear factor $\mathrm{\kappa B}$ in cancer cells versus normal cells. Arch Biochem Biophys 376, 338-346.

45. Tang DG, Chen YQ \& Honn KV (1996) Arachidonate lipoxygenases as essential regulators of cell survival and apoptosis. Proc Natl Acad Sci US A 93, 5241-5246.

46. Winer I, Normolle DP, Shureiqi I, et al. (2002) Expression of 12-lipoxygenase as a biomarker for melanoma carcinogenesis. Melanoma Res 12, 429-434.

47. Greene ER, Huang S, Serhan CN, et al. (2011) Regulation of inflammation in cancer by eicosanoids. Prostaglandins Other Lipid Mediat 96, 27-36.

48. Rhodes LE \& Diffey BL (1996) Quantitative assessment of sunscreen application technique by in vivo fluorescence spectroscopy. J Soc Cosmet Chem 47, 109-115. 